\title{
Repulsive Wnt Signaling Inhibits Axon Regeneration after CNS Injury
}

\author{
Yaobo Liu, ${ }^{1 *}$ Xiaofei Wang, ${ }^{2 *}$ Chin-Chun Lu, ${ }^{1}$ Rachel Sherman-Kermen, ${ }^{1}$ Oswald Steward, ${ }^{3}$ Xiao-Ming Xu, ${ }^{2}$ and Yimin Zou ${ }^{1}$ \\ ${ }^{1}$ Neurobiology Section, Biological Sciences Division, University of California, San Diego, La Jolla, California 92093, ${ }^{2}$ Department of Neurological Surgery, \\ Kentucky Spinal Cord Injury Research Center, University of Louisville School of Medicine, Louisville, Kentucky 40292, and ${ }^{3}$ Reeve-Irvine Center for Neural \\ Regeneration Research, University of California, Irvine, Irvine, California 92697
}

Failure of axon regeneration in the mammalian CNS is attributable in part to the presence of various inhibitory molecules, including myelin-associated proteins and proteoglycans enriched in glial scars. Here, we evaluate whether axon guidance molecules also regulate regenerative growth after injury in adulthood. Wnts are a large family of axon guidance molecules that can attract ascending axons and repel descending axons along the length of the developing spinal cord. Their expression (all 19 Wnts) is not detectable in normal adult spinal cord by in situ hybridization. However, three of them are clearly reinduced after spinal cord injury. Wnt 1 and $W n t 5 a$, encoding potent repellents of the descending corticospinal tract (CST) axons, were robustly and acutely induced broadly in the spinal cord gray matter after unilateral hemisection. Ryk, the conserved repulsive Wnt receptor, was also induced in the lesion area, and Ryk immunoreactivity was found on the lesioned CST axons. Wnt4, which attracts ascending sensory axons in development, was acutely induced in areas closer to the lesion than Wnt1 and Wnt5a. Injection of function-blocking Ryk antibodies into the dorsal bilateral hemisectioned spinal cord either prevented the retraction of CST axons or promoted their regrowth but clearly enhanced the sprouting of CST collateral branches around and beyond the injury site. Therefore, repulsive Wnt signaling may be a cause of cortical spinal tract axon retraction and inhibits axon sprouting after injury.

Key words: Wnt1; Wnt5a; Ryk; spinal cord injury; paralysis; axon retraction; corticospinal tract; collateral branches

\section{Introduction}

Axons in the adult CNS are unable to regenerate after injury, in part, because of the inhibitory molecules in the CNS and lack of growth-stimulating molecules (Yiu and He, 2006). At least some adult CNS axons can regrow when they are presented with peripheral nervous system tissues, such as sciatic nerves (Richardson et al., 1980). This suggests that adult CNS axons can regenerate if the nonpermissive environment is overcome. Two sources of inhibitors have been identified: CNS myelin and the glial scar (Yiu and He, 2006). CNS myelin contains many growthinhibitory molecules [i.e., Nogo, myelin-associated glycoprotein

\footnotetext{
Received Jan. 23, 2008; revised June 19, 2008; accepted June 21, 2008.

This work was supported by National Institute of Neurological Disorders and Stroke Grants R01-NS47484 (Y.Z.), R01-NS36350 and R01-NS52290 (X.-M.X.), and R01-NS047781 (0.S.), and by The Robert Packard Center for ALS Research at The Johns Hopkins University (Y.Z.). Spinal cord injuries in mice were performed in the Roman Reed Core Laboratory at the University of California, Irvine, by Karla Banos. We thank A. Ghosh and members in the Zou Laboratory, C. Lo, L. King, A. Fenstermaker, A. Wolf, A. Lyuksyutova, A. Bechara, and A. Richman for comments and suggests on this manuscript, and Dr. C. Shields and Y.P. Zhang at University of Louisville for providing the VibraKnife device. Patent number 10/847,972 was issued.

*Y.L. and X.W. contributed equally to this work.

Correspondence should be addressed to one of the following: Oswald Steward, Xiao-Ming Xu, or Yimin Zou at the above address. E-mail: osteward@uci.edu, xu26@iupui.edu, oryzou@ucsd.edu.

Y. Liu's present address: Institute of Biophysics, Chinese Academy of Sciences, Beijing 100101, China.

X. Wang's and X.-M. Xu's present address: Spinal Cord and Brain Injury Research Group, Stark Neurosciences Research Institute and Department of Neurological Surgery, Indiana University School of Medicine, Indianapolis, IN 46202.

R. Kerman's present address: The University of Chicago Medical School, Chicago, IL 60637.

DOI:10.1523/JNEUROSCI.1939-08.2008

Copyright $\odot 2008$ Society for Neuroscience $\quad$ 0270-6474/08/288376-07\$15.00/0
}

(MAG), and oligodendrocyte myelin glycoprotein (OMgp)], and chondroitin sulfate proteoglycan (CSPG), enriched in glial scar, is also a potent inhibitor (Bradbury et al., 2002; Curinga et al., 2007). Improvement of regeneration of ascending sensory pathways was observed when signaling pathways leading to inhibition were interrupted by injecting a conventional PKC inhibitor, which blocks all three major species of myelin inhibitors and CSPG. However, a descending pathway, corticospinal tract (CST) axons, failed to regenerate under this treatment (Sivasankaran et al., 2004). Therefore, our understanding of the inhibitory mechanisms in the adult CNS is still rather incomplete. Some axon guidance molecules that function to direct axons during development remain expressed in the adult CNS, whereas others are induced after injury (Miranda et al., 1999; Pasterkamp et al., 1999; De Winter et al., 2002; Bundesen et al., 2003; Goldshmit et al., 2004; Harel and Strittmatter, 2006; Liu et al., 2006). They are candidate regulators of adult axon regeneration.

Wnt proteins are a large family of signaling proteins and control the growth of many axons, including the long tracts in the spinal cord (Zou and Lyuksyutova, 2007). They are critical for the anterior-posterior guidance of spinal cord commissural axons after midline crossing (Lyuksyutova et al., 2003) and posterior growth of CST axons in the dorsal midline of neonatal spinal cord (Liu et al., 2005). We examined the expression of all 19 Wnts and most of their receptors after injury (unilateral hemisection) and found that, although Wnts are normally not expressed at detectable levels in uninjured adult spinal cord, three Wnt genes, Wnt1, 
$W n t 4$, and $W n t 5 a$, are quickly induced surrounding the lesion site after injury. Wntl and Wnt5a are strong repellents of corticospinal tract axons, and Wnt4 does not repel corticospinal tract axons but can attract sensory axons via Frizzled3 (Lyuksyutova et al., 2003; Liu et al., 2005). A repulsive Ryk receptor is acutely induced in the injured corticospinal tract axons. Frizzled 1 is induced around the lesion area and broadly in the cortex, particularly contralateral to the lesion. Injection of function-blocking Ryk antibodies either prevented corticospinal tract axons from retracting or caused significant regrowth after dorsal bilateral hemisection. Function-blocking Ryk antibodies promoted extensive sprouting of collateral branches of CST, around and beyond the lesion site via the uninjured ventral spinal cord. The sprouting beyond the lesion site is either from the dorsal CST bypassing the lesion or from ventral CST. These axon sprouts also cross the midline, as revealed by unilateral biotinylated dextran amine (BDA) tracing.

\section{Materials and Methods}

Spinal cord unilateral hemisection and induction of Wnt signaling system. Spinal cord unilateral hemisection in Figures 1 and $2, A-D$, was performed in adult CD1 mice ( 2 months of age) at C5 level. All procedures used were in compliance with National Institutes of Health guidelines and approved by the Institutional Animal Care and Use Committee of University of California, Irvine. Seven pairs of uninjured and injured mice were analyzed for day 1 , six pairs for day 7 , and seven pairs for day 28. Six pairs of uninjured and injured mice were analyzed for Wnt 1 expression for day 1 ; seven pairs for day 7; six pairs for day 14; six pairs for day 28 for $W n t 5 a$ expression; three pairs for days 1, 7, and 14; and three pairs for Frizzled 1 for days 1, 7, and 14 for Wnt4 expression. At least six pairs of uninjured and injured spinal cords were tested for Ryk protein induction at each time point.

In situ hybridization. In situ hybridization was performed using digoxigenin-labeled sense and antisense RNA probes with alkaline phosphatase detection (Roche Molecular Biochemicals). Specific in situ probes for the entire mouse Wnt gene family (Wnt1, Wnt2, Wnt2b, Wnt3, Wnt3a, Wnt4, Wnt5a, Wnt5b, Wnt6, Wnt7a, Wnt7b, Wnt8a, Wnt8b, Wnt9a, Wnt9b, Wnt10a, Wnt10b, Wnt11, and Wnt16), the Ryk gene and the Frizzled gene family (Frizzled1, Frizzled2, Frizzled3, Frizzled5, Frizzled8, and Frizzled9) were cloned by PCR with reverse transcription (reverse transcription-PCR) from various stages of mouse embryos and adult mouse brains into the pCRII vector (Invitrogen). The in situ probes for Wnt1, Wnt4, Wnt5a, and Ryk have been described previously (Lyuksyutova et al., 2003; Liu et al., 2005). We optimized in situ conditions for adult tissues. The brains and spinal cords were dissected and the spinal cords were cut into 2 -cm-long pieces including whole cervical and most thoracic parts to include the injury site in the center, fixed in $4 \%$ paraformaldehyde, infiltrated with $30 \%$ sucrose, and embedded in OCT (Tissue Tek). Ten micrometer serial horizontal sections of adult spinal cords were cut. Brains were treated in the same condition as spinal cords, and $20 \mu \mathrm{m}$ coronal cortical sections were cut serially along the forebrain in the anterior-to-posterior order. All sections were refixed on slides, treated with $1 \mu \mathrm{g} / \mathrm{ml}$ proteinase $\mathrm{K}$ for $5 \mathrm{~min}$ at room temperature, and hybridized at $56^{\circ} \mathrm{C}$ for $>40 \mathrm{~h}$. Other steps were performed as described previously (Frohman et al., 1990). All probes were applied to screen for mRNA expression in adult spinal cord at different time points (days 1, 7, 14 , and 28) after hemisection, and intact adult spinal cord at the same stage probed side by side as negative control. For each of the postinjury time points examined $(1,7,14$, and $28 \mathrm{~d}$ after lesion), tissue from at least six adult spinal cords and cortices were analyzed.

Immunohistochemistry. Polyclonal anti-Ryk antibody, which we applied in a previous study (Liu et al., 2005), was generated against the ectodomain of Ryk, amino acids 90-183, fused with maltose binding protein, which was purified and injected into rabbits (Hovens et al., 1992). Anti-Ryk antibodies were further purified by protein A-G beads. Both injured and uninjured adult spinal cords were dissected, fixed, and embedded. Ten micrometer serial sections were cut along the horizontal plane. Immunohistochemistry was performed as described previously (Serafini et al., 1996). Uninjured adult spinal cords of the same age were included as negative control. For each of the postinjury time points examined (1, 7, 14, and $28 \mathrm{~d}$ after lesion), tissues from at least six adult spinal cords and cortices were analyzed. Immunostaining with GFAP antibodies was included to mark the lesion sites in Figures 4 and 5. The sections were washed in blocking solution (0.3\% Triton X-100; $10 \%$ normal serum in $0.01 \mathrm{M}$ PBS, $\mathrm{pH}$ 7.4) and were incubated in the primary antibodies diluted in the same blocking solution overnight at $4^{\circ} \mathrm{C}$ followed by fluorescent secondary antibodies for $90 \mathrm{~min}$ at room temperature. The following primary antibodies were used in this study: mouse anti-GFAP (1:400; Invitrogen) or rabbit anti-GFAP (1:200; Millipore Bioscience Research Reagents) to detect glial fibrillary acidic protein; mouse anti-SMI-31 (1:500; Sternberger) to detect axons (in supplemental Fig. 1, available at www.jneurosci.org as supplemental material); mouse anti-growth-associated protein (GAP-43; 1:1000; Millipore Bioscience Research Reagents) to label regenerating axons; mouse anti-ED-1 (1:300; Millipore Bioscience Research Reagents) to microglia/macrophages (in supplemental Fig. 2, available at www.jneurosci.org as supplemental material). The staining was visualized with appropriate secondary antibodies conjugated to TRITC (tetramethylrhodamine isothiocyanate) and FITC (Jackson ImmunoResearch).

Bilateral dorsal funiculotomy and CST tracing and Ryk antibody injection. Adult female SD rats $(210-230 \mathrm{~g} ; n=21)$ were anesthetized with intraperitoneal pentobarbital ( $50 \mathrm{mg} / \mathrm{kg}$; Henry Schein). A partial laminectomy was performed at C3-C4. The dura was cut transversely, and the two edges were pulled rostrally and caudally. A dorsal funiculotomy was performed to a depth of $1.6 \mathrm{~mm}$ from the dorsal surface of the cord and a width of $2.0 \mathrm{~mm}$ using a Vibraknife device (Zhang et al., 2004). Such a lesion bilaterally transects the dorsal funiculi that include the corticospinal and dorsal ascending tracts, as well as the medial portion of the dorsal horn (see Figs. $2 E-Q, 3,4)$. Immediately after lesion, four injections of anti-Ryk antibodies $(100 \mu \mathrm{g} / \mathrm{ml} ; n=11)$ were made into the spinal cord along the dorsal midline at $1.5 \mathrm{~mm}$ rostral, $0.5 \mathrm{~mm}$ rostral, $0.5 \mathrm{~mm}$ caudal, and $1.5 \mathrm{~mm}$ caudal to the lesion site. For each injection site, two injections at depths of 0.6 and $1.0 \mathrm{~mm}$ were made ( $0.5 \mu \mathrm{l}$ at each depth) using a pulled glass capillaries attached to a pneumatic Pico Pump (World Precision Instruments) at a rate of $0.25 \mu \mathrm{l} / \mathrm{min}$. Overlying dura and muscle layers were then sutured and the skin stapled. Injections of artificial CSF (ACSF) $(n=10)$ in identical sites were served as controls. All procedures used were in compliance with National Institutes of Health guidelines and approved by the Animal Care and Use Committee of the University of Louisville School of Medicine.

Three weeks after the first surgery, rats were reanesthetized and stabilized in a stereotaxic frame. After making burr holes over the sensorimotor cortex, BDA (10,000 molecular weight; Invitrogen), an anterograde tracer, was injected into motor cortex as described previously (Sivasankaran et al., 2004). To visualize the longitudinal growth/sprouting of CST axons in sagittal sections (see Fig. 3), BDA injections were made bilaterally. To visualize the growth of CST axons around and beyond the lesion as well as sprouting across the midline in transverse sections (see Fig. 4), BDA injections were made unilaterally.

Two weeks after BDA injection, rats were deeply anesthetized, perfused with $4 \%$ paraformaldehyde, cryoprotected with $30 \%$ sucrose, and sectioned in series either transversely or sagittally at $20 \mu \mathrm{m}$. For BDA reaction, sections were incubated sequentially with $\mathrm{ABC}$ reagent $(1: 250$; $30 \mathrm{~min}$; Vector Laboratories), biotinyl tyramide (1:75; $10 \mathrm{~min}$; PerkinElmer Life Sciences), and extra-avidin FITC (1:500; 2 h; Sigma-Aldrich).

In the experiments to show costaining of Ryk and BDA, we injected $\mathrm{BDA}$ before the lesion to allow BDA to diffuse along the CST so that when we analyzed CSTs shortly after lesion, they were already labeled (see Fig. $2 E-P$ ). One week after BDA injection, the bilateral dorsal hemisection was performed as described previously (see Fig. $2 E-P$ ). One day or $7 \mathrm{~d}$ after the surgery, spinal cords were dissected for analyses.

Quantification of BDA-labeled CST axons. In all cases, quantification was performed with the experimenter blind to the treatment group (see Figs. 3, 4). For quantification of BDA-labeled CST axons shown in Figure 3 , three sagittal sections of the spinal cord spaced $60 \mu \mathrm{m}$ apart were chosen. The first section was cut along the midline where the central 
canal was visualized. The second section is 60 $\mu \mathrm{m}$ and the third $120 \mu \mathrm{m}$ away from the midline on the same side. The number of BDApositive CST axons is presented as CST axon index at $-1,-0.5,-0.25,-0.1,0,0.5$, and 1 $\mathrm{mm}$ positions, relative to the lesion center, which is indicated as " 0 " (see Fig. $3 E$ ). Axon index is a ratio of the $\mathrm{BDA}^{+}$axon number at a specific position over the axon number $3 \mathrm{~mm}$ rostral to lesion (100\% for the $3 \mathrm{~mm}$ position) (Cafferty et al., 2007). For quantification of $\mathrm{BDA}^{+}$axons in Figure 4, two transverse sections at each of six spinal levels [i.e., C2, C3, C4 $(1.0 \mathrm{~mm}$ rostral, lesion epicenter, and $1.0 \mathrm{~mm}$ caudal), and C5] were analyzed and averaged. Pixel threshold of BDA labeling was determined within the defined regions using Scion Image software. The thresholds for positive staining were set, and the number of pixels above threshold was calculated. Pixels of ventral CST (vCST) and dorsolateral CST (dlCST) were subtracted from calculation. Nonspecific labeling of objects other than axons, such as artificial spots resulting from tissue processing, were also excluded from calculation.

Statistical analyses. Using SPSS software, data were analyzed by one-way ANOVA followed by Dunnett's post hoc tests of multiple comparisons to determine whether there were significant differences between individual groups. Statistical significance was established when $p<0.05$.

\section{Results}

\section{Rapid induction of Wnts after spinal} cord injury

To investigate the role of Wnts in regulating axon regeneration after spinal cord injury, we examined the expression of all Wnt genes over a period of 1 month after spinal cord hemisection at the cervical level, C5, in mice (Fig. $1 M$ ). The uninjured adult spinal cord does not express any of the 19 members (Fig. $1 B, D, G)$ (data not shown). However, we found that a small number of Wnt genes were induced after injury. Wnt1 and $W n t 5 a$, potent repellents of developing corticospinal tract axons, were rapidly and robustly induced broadly both rostral and caudal to the lesion area $1 \mathrm{~d}$ after injury (Fig. $1 A, C$ ). Interestingly, Wnt1 and $W n t 5 a$ are also induced in the areas on the contralateral side of the injury site. These cells that express induced Wnts on the contralateral side may be neurons that send axon across the midline because many of them are NeuNpositive (data not shown). Alternatively, they may be neurons that are connected to those whose axons are severed. The induced Wnt expression is robust and dynamic. Strong induction appears rapidly $1 \mathrm{~d}$ after injury and persists for $7 \mathrm{~d}$. Expression starts to decline after 1 week, and by $28 \mathrm{~d}$, the levels of $W n t 1$ and $W n t 5 a$ mRNA decreased to background level (Fig. $1 N$ ). In addition to Wnt1 and $W n t 5 a$, which encode potent repellents for corticospinal tract axons,
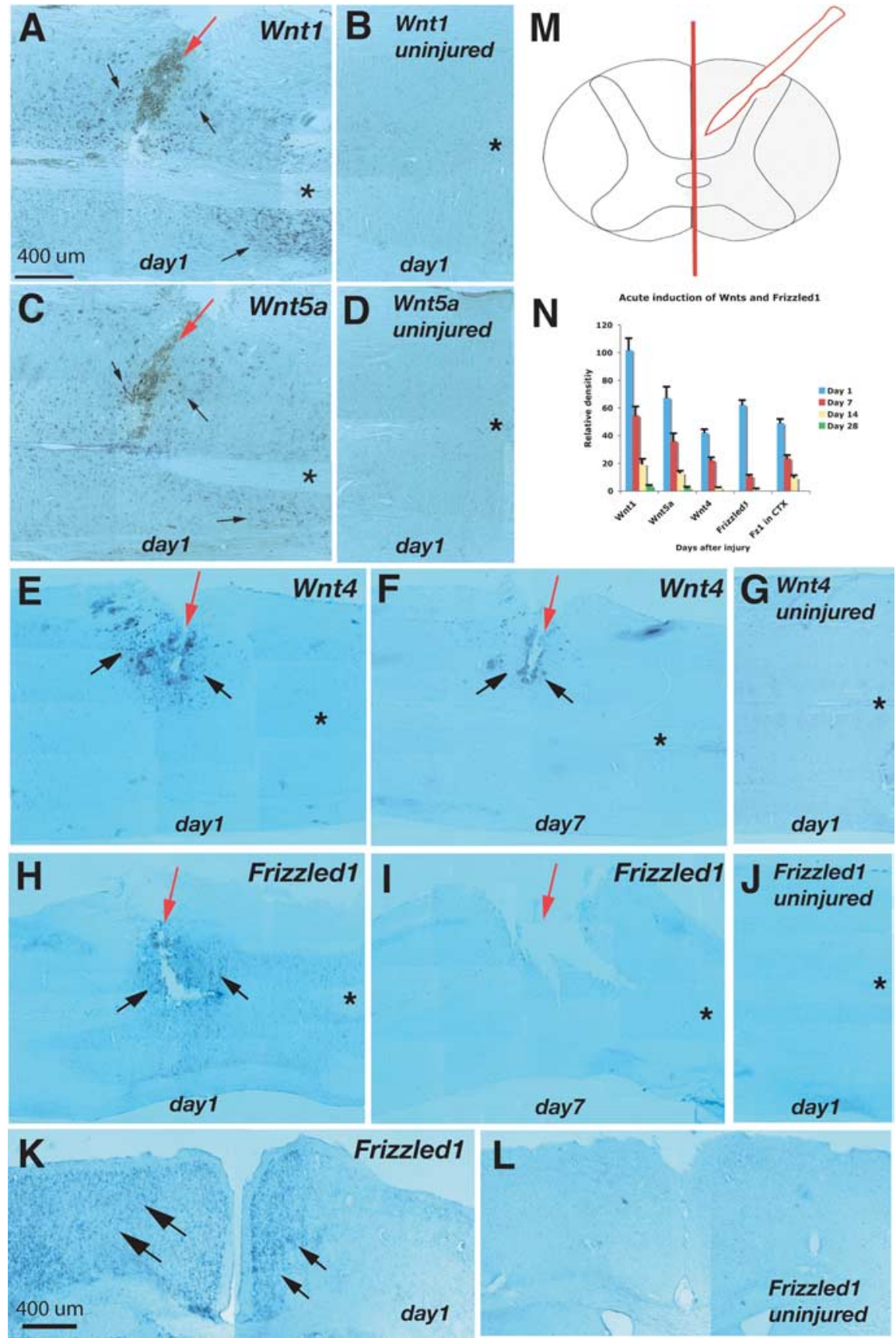

Figure 1. Acute induction of Wnt1, Wnt4, Wnt5, and Frizzled 1 in adult spinal cord gray matter and the cortex after lateral hemisection. A-J, Induction of Wnt1, Wnt4, Wnt5a, and Frizzled $1 \mathrm{mRNA}$ in injured adult spinal cord. All sections are horizontal sections with rostral to the left and caudal to the right. The red arrows indicate the location of lateral hemisection. The black arrows indicate the induced mRNA detected by in situ hybridization. The asterisks indicate the positions of the midline. $K, L$, Rapid induction of Frizzled 1 transcripts in the cortex of the injured mice. $M$, Diagram showing unilateral hemisection of an adult spinal cord. Complete lesion was made on one side of the spinal cord. $N$, Quantification of in situ signals at different time points after injury. For Wnt 1 expression, seven pairs of uninjured and injured mice were analyzed for day 1 , six pairs for day 7 , seven pairs for day 14 , and seven pairs for day 28 . For Wnt5a, six pairs of uninjured and injured mice were analyzed for day 1 , seven pairs for day 7 , six pairs for day 14 , and six pairs for day 28 . Three pairs of days 1,7, and 14 were analyzed for Wnt 4 and Frizzled1. CTX, Cortex. Error bars indicate SEM. Scale bars, $400 \mu \mathrm{m}$.

we also observed rapid induction of Wnt4 in the gray matter surrounding the lesion, which decreases in levels within a week, much sooner than the decline of Wnt1 and Wnt5a (Fig. $1 E, F)$. Wnt4 does not appear to repel corticospinal tract axons (data not shown) (Liu et al., 2005). Therefore, the induced Wnt4 is unlikely responsible for regeneration inhibition but may be responsible for axonal sprouting instead. Frizzled 1 was 

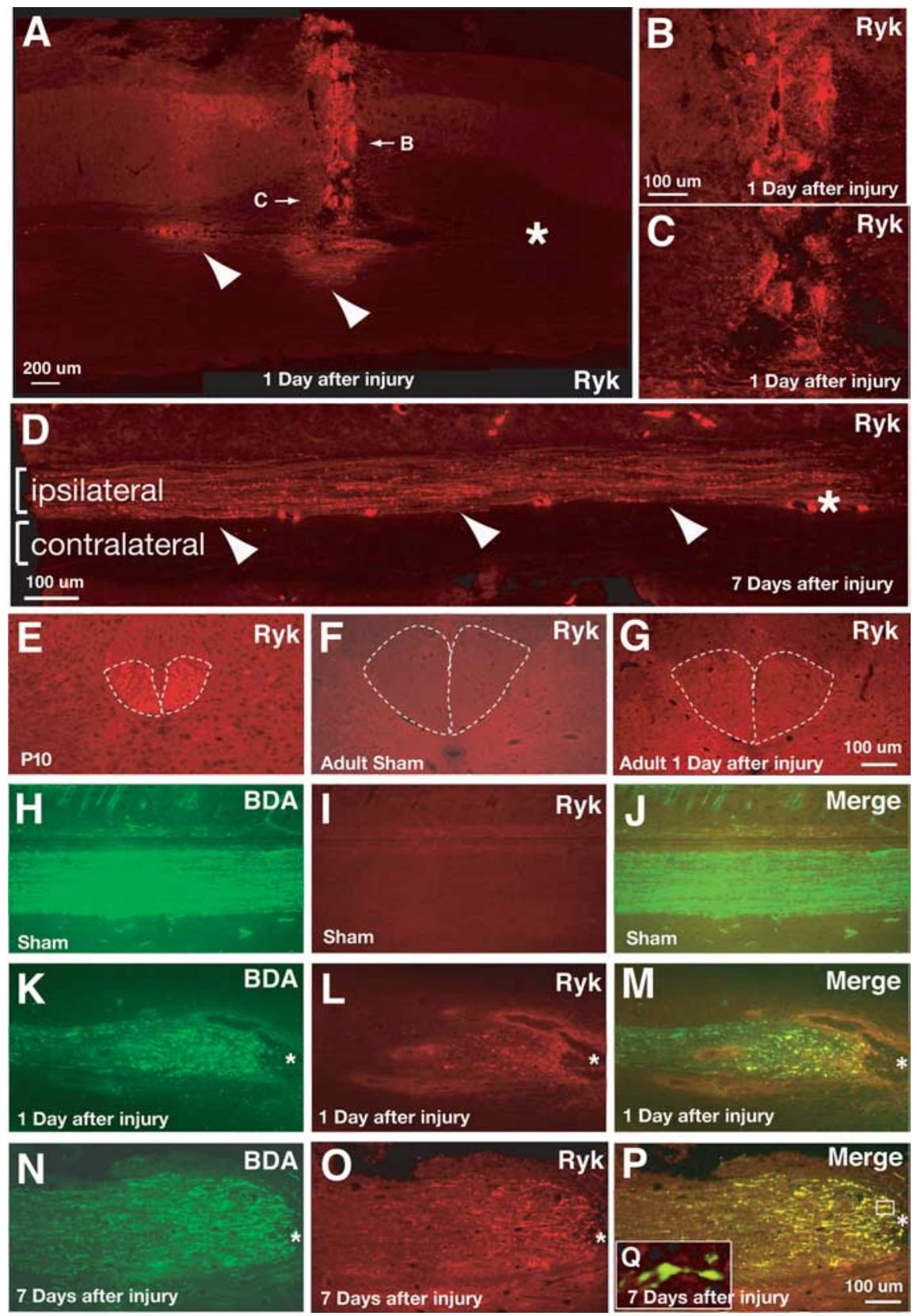

Figure 2. Induction of a repulsive Wnt receptor Ryk on injured corticospinal tract axons. A, Immunostaining with anti-Ryk antibodies of adult spinal cord (horizontal sections) $1 \mathrm{~d}$ after lateral hemisection. Ryk immunoreactivity in the lesion area (labeled $B$ and $C$ ) as well as white matter tracts at the midline (white asterisk) rostral to the lesion site (white arrowheads) was observed. $\boldsymbol{B}, \boldsymbol{C}$, High magnification of areas indicated in A. D, Immunostaining with Ryk antibodies of adult spinal cord horizontal section (5 $\mu \mathrm{m}) 7 \mathrm{~d}$ after hemisection. Note the Ryk-positive staining in the dorsal funiculus ipsilateral to the lesion but not contralateral to the lesion. $\boldsymbol{E}-\mathbf{G}$, immunostaining of transverse sections of the dorsal funiculus with Ryk antibodies. Ryk antibodies stain the CST at P10 $(\boldsymbol{E})$ but not in adult CST $(\boldsymbol{F})$. Ryk immunoreactivity was detected as early as $1 \mathrm{~d}$ after injury of adult spinal cord in the CST area (bilateral dorsal hemisection) (G). $\mathbf{H}-\mathbf{Q}$, Costaining with Ryk antibodies and BDA traced from the motor cortex shows that Ryk protein is induced at the tip of the CST axons shortly after lesion and right at the lesion site. Large retraction bulbs costained with Ryk and BDA were observed ( $\boldsymbol{Q}$ ). Sections are horizontal. Lesion was bilateral dorsal hemisection. Scale bars: $\boldsymbol{A}, 200 \mu \mathrm{m} ; \boldsymbol{B}-\boldsymbol{D}, 100$ $\mu \mathrm{m} ; \boldsymbol{E}-\boldsymbol{P}, 100 \mu \mathrm{m}$. The asterisks in $\boldsymbol{A}-\boldsymbol{D}$ indicate the midline. The asterisks in $\boldsymbol{K}-\boldsymbol{P}$ indicate the location of the dorsal hemisection lesion.

found induced in similar regions immediately around the lesion (Fig. $1 H$ ) and declined at a similar rate as Wnt4 (Fig. 1I). Frizzled 1 mRNA is also rapidly and broadly induced in the cortex, particularly in the contralateral side to the injury, and declined within a week (Fig. $1 K, N$ ). Neither Wnt4 nor Frizzled 1 are expressed in uninjured spinal adult spinal cord (Fig. 1G,J) or cortex (Fig. 1L).
Acute induction of a repulsive Wnt receptor, Ryk, in corticospinal tract axons

Ryk, a Wnt receptor that mediates repulsive function of Wnt proteins, was found to be induced acutely after injury. Ryk protein is expressed in developing corticospinal tract axons during the first 2 postnatal weeks (Liu et al., 2005). As shown in Figure $2 E$, Ryk antibodies label the ventral most part of the dorsal funiculus, where the rodent CST axons are located in transverse sections of postnatal day 10 rat. In adult spinal cord, Ryk is no longer expressed throughout the spinal cord. No staining in the dorsal funiculus is seen in uninjured spinal cord (Fig. 2F). However, Ryk protein was observed at the lesion site acutely and can be clearly detected $1 \mathrm{~d}$ after injury using polyclonal antibodies against Ryk developed in the laboratory (Fig. $2 A-C, G$ ) (Liu et al., 2005). Interestingly, Ryk protein was abundant in the white matter tracts in the midline anterior and adjacent to the lesion area in the horizontal plane (Fig. $2 A, D$, arrowheads). These axon tracts may be descending CST axons judged by the position of these axons. Signals of Ryk in situ hybridization are typically weak, probably because of the low levels of mRNA attributable to either low expression level or stability of mRNA (Liu et al., 2005). But Ryk mRNA can be detected in the correct layer in the motor cortex contralateral to the spinal cord lesion $24 \mathrm{~h}$ after injury (data not shown). To further confirm that the Ryk protein was induced in CST axons after spinal cord injury, we injected the anterograde tracer BDA into the frontal motor cortex and found that the white matter fibers in which Ryk was induced did originate from the motor cortex, because they are also labeled positively by the $\mathrm{BDA}$ tracer (Fig. $2 \mathrm{~K}-\mathrm{P}$ ). We performed the coimmunostaining at higher resolution at the lesion site after dorsal hemisection. After dorsal hemisection, Ryk immunoreactivity was quickly observed in the CST axons rostral to the lesion (Fig. $2 G$ in transverse section $1 \mathrm{~mm}$ rostral to the lesion; Fig. $2 \mathrm{~L}, \mathrm{O}$ in horizontal sections). We found that Ryk and BDA staining perfectly colocalize in the injured axons (Fig. $2 M, P$, yellow) and at the tip of the injured axons in the large retraction bulbs (Fig. 2Q). The expression of Ryk can be detected as early as day 1 but is much stronger at day 7 (Fig. 2L,O). Therefore, a repulsive Wnt receptor, Ryk, is acutely induced in the CST axons after injury.

\section{Function-blocking Ryk antibodies prevented CST axon retraction after injury}

The polyclonal Ryk antibodies are function blocking and can neutralize the repulsive function of Wnt1 and Wnt5a of CST axons in vivo and corpus callosum axons in culture (Liu et al., 
2005; Keeble et al., 2006). To address whether the injury-induced Wnts may play a role in blocking CST regeneration in vivo, we injected purified anti-Ryk antibodies (dialyzed into artificial corticospinal fluid) into lesioned adult rat spinal cord. A bilateral dorsal funiculotomy was performed at the $\mathrm{C} 4$ level, which completely transected the bilateral dorsal funiculi that contain the corticospinal tracts (Iannotti et al., 2006). Anti-Ryk antibodies $(100 \mu \mathrm{g} / \mathrm{ml} ; n=11)$ were injected into the spinal cord at 1.5 rostral, $0.5 \mathrm{~mm}$ rostral, 0.5 caudal, and $1.5 \mathrm{~mm}$ caudal to the lesion along the midline. To visualize the longitudinal growth of CST axons, the BDA injections were made bilaterally and sagittal sections were analyzed. We found that 5 weeks after the initial lesion, in control animals injected with artificial corticospinal fluid, many corticospinal tract axons retracted up to $1 \mathrm{~mm}$ away from the lesion border as shown by BDA axon tracing (Fig. $3 A, B)$. GFAP staining marks the border of the lesion. However, in the Ryk antibodies-injected animals, the presence of many CST axons close to the lesion border was observed as revealed by BDA tracing (Fig. 3C,D). The difference in the number of BDA-labeled CST axons in regions immediately rostral to the injury $(<1 \mathrm{~mm})$ was statistically significant (Fig. $3 E$ ). Notably, the retraction bulbs, frequently seen after corticospinal tractotomy, were markedly reduced in both the size and number after the anti-Ryk treatment (Fig. 3D) compared with the ACSF treatment (Fig. 3B).

\section{Function-blocking Ryk antibodies caused sprouting of CST collateral branches after injury}

We also observed sprouting of CST collateral branches when Ryk antibodies were injected. We divided spinal cord into four zones (Fig. 4A). Zone I is the lateral two-thirds of the contralateral area, mainly the gray matter. Zone II is the inner one-third of the contralateral area, including the location of the unlabeled corticospinal tract (unilaterally labeled) and the medial one-third of the contralateral gray matter. Zone III is the inner one-third of the lesioned side that contains the ipsilaterally labeled corticospinal tract and medial one-third of ipsilateral gray matter. Zone IV is the outer two-thirds of the ipsilateral gray matter that contains labeled terminals branched off from the main corticospinal tract. We found that anti-Ryk treatment promoted substantial sprouting of CST axons rostral to the injury (Fig. $4 \mathrm{~J}$, double arrows), in the gray matter ventral to the lesion (Fig. $4 K$, arrows) and caudal to the lesion (Fig. $4 L$, arrows). These branches found beyond the lesion site could be caused by nascent branches from dorsal CST bypassing the lesion site via the intact ventral spinal cord tissue or by sprouting from the ventral CST. Moreover, BDA-labeled CST axons were also found to cross the midline to innervate the contralateral gray matter (Fig. $4 K, L$, arrowheads). In contrast, ACSF treatment did not induce significant CST axonal sprouting in areas rostral, at, or caudal to the
ACSF

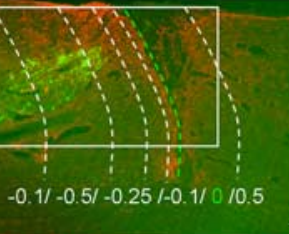

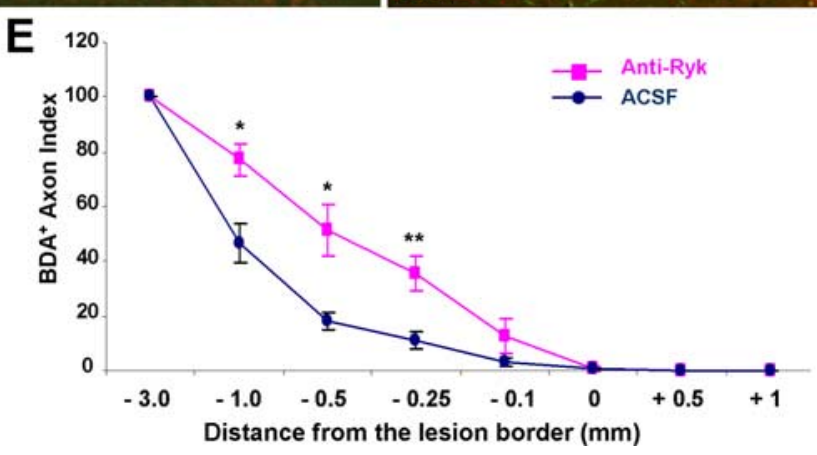

Anti-Ryk
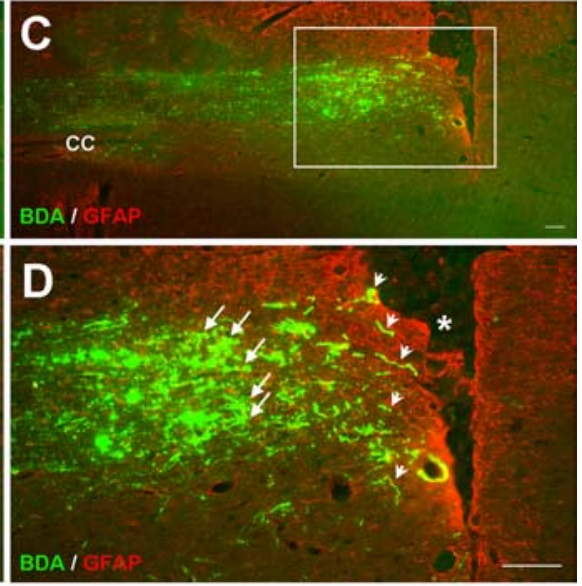

Figure 3. Anti-Ryk antibodies inhibited retraction and dying back of CST axons after bilateral dorsal funiculotomy. $\boldsymbol{A}$, In a 列 rea in (clearly illustrates the presence of CST fibers (short arrow) up to the lesion border (red dashed line) indicating that either retraction of CST axons was prevented or CST axons regrew back to the lesion border. Noticeably, the size and number of retraction confirmed the presence of significantly more axons within $1 \mathrm{~mm}$ proximal to the lesion border in the anti-Ryk-treated group compared with the control group $\left(n=5\right.$; mean \pm SEM; $\left.{ }^{*} p<0.05,{ }^{* *} p<0.01\right)$. CC, Central canal. Asterisks in $\boldsymbol{B}$ and $\boldsymbol{D}$ indicate the lesion. Scale bars: $\boldsymbol{A}, \boldsymbol{C}, 150 \mu \mathrm{m} ; \boldsymbol{B}, \boldsymbol{D}, 300 \mu \mathrm{m}$.

injury (Fig. 4B-G). Quantification of BDA-labeled CST axons in zones I-IV further confirmed a significant increase in the number of CST fibers in areas rostral, at, and caudal to the injury (Fig. 4P, Q) as well as in the contralateral gray matter at and caudal to the injury (Fig. 4O). It should be noted that the sprouting of adult regenerating CST collateral branches to the contralateral gray matter after Ryk antibody injection is reminiscent of the reported response of young CST axons when lesion was introduced right after birth (Bregman et al., 1989). We did not observe CST axons within the lesion site (judging by the lack of BDA signals), although GAP-43-positive and SMI31-positive regenerating axons of a currently unknown source were observed within the lesion site (supplemental Fig. 1, available at www.jneurosci.org as supplemental material). We found that antibody treatment did not cause a significant change of the ED-1-positive cells at the lesion area (supplemental Fig. 2, available at www.jneurosci.org as supplemental material).

\section{Discussion}

We report that the Wnt axon guidance system is robustly and acutely reinduced in the injured CNS and plays an important role 


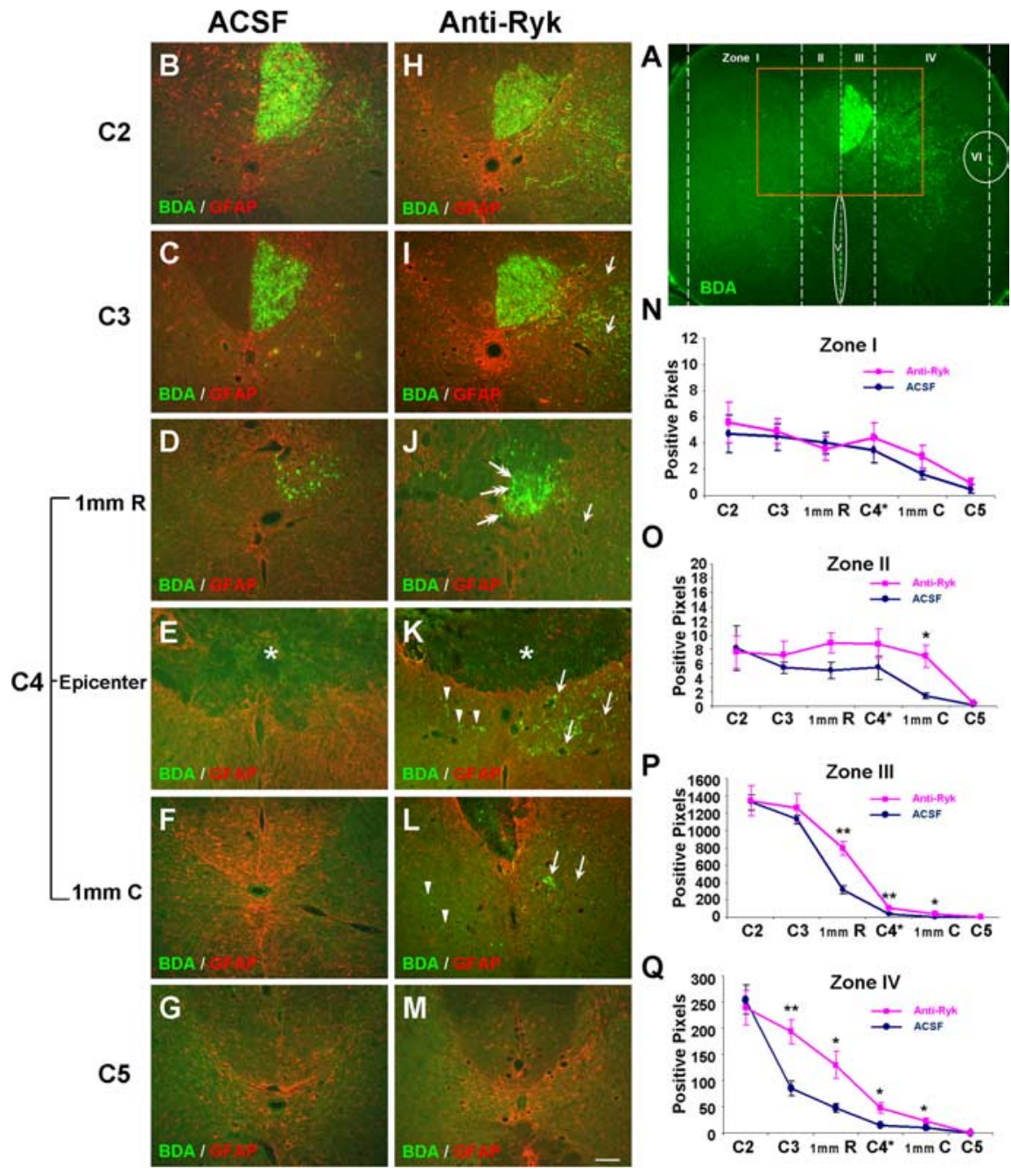

Figure 4. Anti-Ryk antibodies induced sprouting of CST axons around and beyond the site of dorsal funiculotomy. $A, A$ transverse section of a C 4 cervical spinal cord shows a unilaterally labeled dorsal CST located in the ventral portion of the dorsal funiculus and its collateral projection to the unilateral lateral and ventral gray matter. To quantify the labeling intensity of CST axons, the spinal cord cross section was divided into zones I-IV, demarcated by dashed lines. To avoid the misinterpretation of data, labeled fibers in regions of the vCST (oval) and dICST (circle) were excluded from quantification. $\boldsymbol{B}-\boldsymbol{M}$, High magnification of spinal cord section areas, demarcated in $\boldsymbol{A}$ (red), showed patterns of CST labeling rostral (C2, C3), at (C4, $1 \mathrm{~mm}$ rostral and caudal), and caudal (C5) to the injury (asterisk) between the control (ACSF; left column) and anti-Rky-treated (right column) groups. Anti-Ryk antibody treatment promoted substantial sprouting of CST axons within its original pathway rostral to the injury $(J)$ (double arrow), into the gray matter (arrow) ventral to the lesion $(\boldsymbol{K})$ (asterisk), and descended caudal to the injury $(\boldsymbol{L})$ (arrow). Moreover, at and below the level of injury, sprouting of labeled CST axons across the midline was found $(\boldsymbol{K}, \boldsymbol{L})$ (arrowhead). In contrast, CST axonal sprouting rostral, at, and caudal to the lesion site was not found in the control group treated with ACSF $(\boldsymbol{B}-\boldsymbol{G})$. At the $C 5$ level, no labeled CST axons were found in both groups $(\boldsymbol{G}, \boldsymbol{M}) . \mathbf{N}-\mathbf{Q}$, Quantification of BDA-labeled CST axons in zones I-IV revealed a significant increase in the amount of CST labeling rostral to the injury (zone III in $P$ ), in the ipsilateral gray matter rostral, at, and caudal to the injury (zone IV in $\mathbf{Q}$ ), and in the contralateral gray matter caudal to the injury (zone II in $\mathbf{0}$ ) in the anti-Ryk-treated group compared with the control group. ( $n=5$ ASCF group; $n=6$ anti-Ryk group; mean \pm SEM of positive axon pixels; ${ }^{*} p<0.05,{ }^{* *} p<0.01$ ). Scale bar, $200 \mu \mathrm{m}$.

in regulating regeneration of at least CST axons after spinal cord injury. After examining the expression pattern of the 19 members of the Wnt family in adult mouse spinal cord, we found that two potent CST repellents, encoded by $W n t 1$ and $W n t 5$, are rapidly and broadly induced in the gray matter of the adult spinal cord after hemisection. The expression is dynamic and declines within 1 week. One known axon attractant, Wnt4, was also induced close to the lesion but declined more rapidly than Wnt1 and Wnt5a. We also observed that Ryk, a repulsive Wnt receptor, was induced on the injured corticospinal tract axons as confirmed by BDA tracing. The reinduced Ryk increases from days 1 to 7 . Frizzled receptors undergo dynamic changes as well, with Frizzled1 being reinduced in the spinal cord and the motor cortex. Function-blocking antibodies against Ryk either prevented retraction of CST axons or promoted regrowth because many CST axon terminals were found right at the border of the lesion site, in stark contrast to the ACSF control-injected injured animals in which axons were found retracted far away from the lesion site. We currently cannot distinguish whether CST axons never underwent retraction or they first retracted and then grew back after Ryk antibody injection. Future studies with careful time course analyses after injury and injection will be needed. However, Ryk antibodies injection clearly promoted the regenerative growth of CST collateral fibers around and beyond the injury site. Because we did not see axons directly traversing the lesion, the axon sprouts that extended past the lesion were not spared axons and should represent newly grown axons. Together, these results suggest that the Wnt axon guidance systems may be reinduced in the adult CNS after injury, and overcoming the Wnt inhibition will likely improve regeneration. Ryk is a highly unique protein in the CNS and not a member of a large protein family, making it an excellent specific therapeutic target for promoting regeneration.

Because no antibodies against Wnt or Wnt5a work effectively in immunostaining, we are currently unable to pinpoint which cell types express the induced Wnts or Frizzled1. We performed a crude immunohistochemistry and in situ hybridization on consecutive slides and overlaid the sections to determine the cell types. The results appear to suggest that Wnts are induced in multiple cell types, including the NeuN-positive neurons, NG-2-positive cells, and ED-1-positive cells macrophage (data not shown). Because Wnts are secreted proteins, regardless of the cell types expressing the proteins, the injured spinal cord close to the lesioned areas may contain Wnt proteins.

Axon regeneration in the CNS is blocked by multiple sources of inhibitors. The central myelin and the glial scar are well known for producing inhibitors such as the myelin-associated molecules, Nogo, MAG, and OMgp, and CSPGs on astroglial scar (Yiu and He, 2006). Some improvement of regeneration is already shown when some of these inhibitors are blocked, particular by modifying the CSPGs with the enzyme ChABC (chondroitinase ABC) (Bradbury et al., 2002; Curinga et al., 2007). However, the incomplete regeneration may very well be attributable to additional inhibitors, especially in the case of corticospinal tract axons. In recent years, several axon guidance molecules and their receptors, such as members of ephrin and Semaphorin families, were found 
induced close to or within lesion sites after adult CNS injury (Miranda et al., 1999; Pasterkamp et al., 1999; De Winter et al., 2002; Bundesen et al., 2003; Goldshmit et al., 2004; Liu et al., 2006). It is currently unknown whether these reinduced axon guidance systems play a direct role in regulating the regenerative growth of axons after traumatic injury. However, EphB regulates astrocyte gliosis and formation of glial scar, and therefore EphB/ ephrinB signaling may be important regulators of regeneration (Du et al., 2007). We show here that the Wnt family of proteins and their receptors are also quickly induced at the lesion and on lesioned axons, respectively, and may provide strong inhibition/repulsion to CNS axons, such as CST axons. It should be noted that after Ryk antibody treatment, many CST axons were present right at the border of the lesion created by VibraKnife. Although the lesion gap was filled with cells and we observed Neurofilament-positive and GAP-43-positive and SMI-31-positive axons in the lesion site, no BDA-positive CST axons were present inside the lesion (supplemental Fig. 1, available at www.jneurosci.org as supplemental material). It is possible that additional inhibitors within the lesion site/scar tissue, such as Sema3A and CSPG, prevent axons from regenerating across the lesion. Therefore, a combinatorial approach targeting different inhibitory systems will likely yield greater results. However, our results suggest that Wnts may be mainly responsible for the massive axonal retraction after injury. Overcoming/reversing retraction will be an important component in efforts of axon regeneration after traumatic injury.

Intriguingly, Frizzled receptors, which are known to mediate axon attraction in vertebrates and flies, are also reinduced after injury (Lyuksyutova et al., 2003; Sato et al., 2006; Wolf et al., 2008). It is plausible that the reinduced Frizzled may be responsible for corticospinal tract axons sprouting, which is then quickly inhibited by the reinduced Ryk, a higher-affinity Wnt receptor than Frizzleds (Liu et al., 2005; Schmitt et al., 2006). Consistent with this, the expression of induced Frizzled 1 declines sooner than $R y k$. The enhanced sprouting when Ryk function is blocked may reflect the growth-promoting function of WntFrizzled signaling. Therefore, enhancing Wnt-Frizzled signaling for attraction (Wolf et al., 2008) and, in the meantime, inhibiting Wnt-Ryk repulsion may result in more robust regeneration and provide effective supplement to other methods of promoting regeneration. It will be interesting to test the potential role of Frizzleds in injury and regeneration.

\section{References}

Bradbury EJ, Moon LD, Popat RJ, King VR, Bennett GS, Patel PN, Fawcett JW, McMahon SB (2002) Chondroitinase ABC promotes functional recovery after spinal cord injury. Nature 416:636-640.

Bregman BS, Kunkel-Bagden E, McAtee M, O’Neill A (1989) Extension of the critical period for developmental plasticity of the corticospinal pathway. J Comp Neurol 282:355-370.

Bundesen LQ, Scheel TA, Bregman BS, Kromer LF (2003) Ephrin-B2 and EphB2 regulation of astrocyte-meningeal fibroblast interactions in response to spinal cord lesions in adult rats. J Neurosci 23:7789-7800.

Cafferty WB, Yang SH, Duffy PJ, Li S, Strittmatter SM (2007) Functional axonal regeneration through astrocytic scar genetically modified to digest chondroitin sulfate proteoglycans. J Neurosci 27:2176-2185.

Curinga GM, Snow DM, Mashburn C, Kohler K, Thobaben R, Caggiano AO, Smith GM (2007) Mammalian-produced chondroitinase AC mitigates axon inhibition by chondroitin sulfate proteoglycans. J Neurochem 102:275-288.

De Winter F, Oudega M, Lankhorst AJ, Hamers FP, Blits B, Ruitenberg MJ, Pasterkamp RJ, Gispen WH, Verhaagen J (2002) Injury-induced class 3 semaphorin expression in the rat spinal cord. Exp Neurol 175:61-75.
Du J, Fu C, Sretavan DW (2007) Eph/ephrin signaling as a potential therapeutic target after central nervous system injury. Curr Pharm Des 13:2507-2518.

Frohman MA, Boyle M, Martin GR (1990) Isolation of the mouse Hox-2.9 gene; analysis of embryonic expression suggests that positional information along the anterior-posterior axis is specified by mesoderm. Development 110:589-607.

Goldshmit Y, Galea MP, Wise G, Bartlett PF, Turnley AM (2004) Axonal regeneration and lack of astrocytic gliosis in EphA4-deficient mice. J Neurosci 24:10064-10073.

Harel NY, Strittmatter SM (2006) Can regenerating axons recapitulate developmental guidance during recovery from spinal cord injury? Nat Rev Neurosci 7:603-616.

Hovens CM, Stacker SA, Andres AC, Harpur AG, Ziemiecki A, Wilks AF (1992) RYK, a receptor tyrosine kinase-related molecule with unusual kinase domain motifs. Proc Natl Acad Sci U S A 89:11818-11822.

Iannotti C, Zhang YP, Shields LB, Han Y, Burke DA, Xu XM, Shields CB (2006) Dural repair reduces connective tissue scar invasion and cystic cavity formation after acute spinal cord laceration injury in adult rats. J Neurotrauma 23:853-865.

Keeble TR, Halford MM, Seaman C, Kee N, Macheda M, Anderson RB, Stacker SA, Cooper HM (2006) The Wnt receptor Ryk is required for Wnt5a-mediated axon guidance on the contralateral side of the corpus callosum. J Neurosci 26:5840-5848.

Liu X, Hawkes E, Ishimaru T, Tran T, Sretavan DW (2006) EphB3: an endogenous mediator of adult axonal plasticity and regrowth after CNS injury. J Neurosci 26:3087-3101.

Liu Y, Shi J, Lu CC, Wang ZB, Lyuksyutova AI, Song XJ, Song X, Zou Y (2005) Ryk-mediated Wnt repulsion regulates posterior-directed growth of corticospinal tract. Nat Neurosci 8:1151-1159.

Lyuksyutova AI, Lu CC, Milanesio N, King LA, Guo N, Wang Y, Nathans J, Tessier-Lavigne M, Zou Y (2003) Anterior-posterior guidance of commissural axons by Wnt-frizzled signaling. Science 302:1984-1988.

Miranda JD, White LA, Marcillo AE, Willson CA, Jagid J, Whittemore SR (1999) Induction of Eph B3 after spinal cord injury. Exp Neurol 156:218-222

Pasterkamp RJ, Giger RJ, Ruitenberg MJ, Holtmaat AJ, De Wit J, De Winter F, Verhaagen J (1999) Expression of the gene encoding the chemorepellent semaphorin III is induced in the fibroblast component of neural scar tissue formed following injuries of adult but not neonatal CNS. Mol Cell Neurosci 13:143-166.

Richardson PM, McGuinness UM, Aguayo AJ (1980) Axons from CNS neurons regenerate into PNS grafts. Nature 284:264-265.

Sato M, Umetsu D, Murakami S, Yasugi T, Tabata T (2006) DWnt4 regulates the dorsoventral specificity of retinal projections in the Drosophila melanogaster visual system. Nat Neurosci 9:67-75.

Schmitt AM, Shi J, Wolf AM, Lu CC, King LA, Zou Y (2006) Wnt-Ryk signalling mediates medial-lateral retinotectal topographic mapping. $\mathrm{Na}-$ ture 439:31-37.

Serafini T, Colamarino SA, Leonardo ED, Wang H, Beddington R, Skarnes WC, Tessier-Lavigne M (1996) Netrin-1 is required for commissural axon guidance in the developing vertebrate nervous system. Cell 87:1001-1014.

Sivasankaran R, Pei J, Wang KC, Zhang YP, Shields CB, Xu XM, He Z (2004) PKC mediates inhibitory effects of myelin and chondroitin sulfate proteoglycans on axonal regeneration. Nat Neurosci 7:261-268.

Wolf AM, Lyuksyutova AI, Fenstermaker AG, Shafer B, Lo CG, Zou Y (2008) Phosphatidylinositol-3-kinase-atypical protein kinase C signaling is required for Wnt attraction and anterior-posterior axon guidance. J Neurosci 28:3456-3467.

Yiu G, He Z (2006) Glial inhibition of CNS axon regeneration. Nat Rev Neurosci 7:617-627.

Zhang YP, Iannotti C, Shields LB, Han Y, Burke DA, Xu XM, Shields CB (2004) Dural closure, cord approximation, and clot removal: enhancement of tissue sparing in a novel laceration spinal cord injury model. J Neurosurg 100:343-352.

Zou Y, Lyuksyutova AI (2007) Morphogens as conserved axon guidance cues. Curr Opin Neurobiol 17:22-28. 